\title{
Fabrication and Evaluation of Sustained Release Mucoadhesive Losartan Potassium Matrix Tablets
}

\author{
Md. Emdadul Hasan Mukul, Sayed Koushik Ahmed, Mariyum Akter and \\ Mohammad Salim Hossain
}

Department of Pharmacy, Noakhali Science and Technology University Sonapur, Noakhali-3814, Bangladesh

\begin{abstract}
Sustained release matrix tablets of Losartan potassium were prepared with different types of mucoadhesive polymers e.g. Hydroxypropylmethyl cellulose (HPMC), Sodium carboxymethyl cellulose (Na-CMC) and Methyl cellulose (MC). The tablets were prepared by direct compression method and then were subjected to in-vitro drug dissolution for 8 hours in phosphate buffer of $\mathrm{pH}$ 6.8. The mucoadhesive property was investigated in terms of retention time by wash-off method. Irrespective of amount of polymer, mucoadhesive strength of MC was proved to be maximum where HPMC and Na-CMC showed moderate or poor mucoadhesive strength. Drug release from all formulations followed Higuchi release mechanism.
\end{abstract}

Key word: Mucoadhesion, Losartan Potassium, Retention time, Release kinetics.

\section{Introduction}

Losartan potassium is an angiotensin II receptor antagonist drug used mainly to treat high blood pressure (hypertension). Orally administered dosage form shows some problems such as too short gastric retention (GRT) and unpredictable gastric emptying-time. These problems can be overcome by altering the gastric emptying which is affected by age, sex, and health condition. It is therefore desirable to formulate a sustained release dosage form that gives an extended GI residence time (Remington, 2005).

Various approaches have been worked out to improve the retention of an oral dosage form in the stomach including mucoadhesive systems (Vyas et al., 2002). Mucoadhesive systems are used to improve the drug absorption process in site-specific manner (Wilson et al., 1989). At physiological $\mathrm{pH}$ the mucin network has a net negative charge due to the presence of sialic acid having $\mathrm{p}^{\mathrm{Ka}} 2.6$ and presence of sulphate residue (Schachter et al.,1982). Mucous is a network of mucin glycoproteins. Bioadhesive polymers having hydrophilic functional groups (e.g. carboxyl, hydroxyl, amide and sulphate groups) which form hydrogen bond with sialic acid that present in mucous membrane. These polymers also form viscous layers when hydrated with water that increases the retention time (Hannah, 2004). Losartan potassium was used as a model drug to develop, evaluate and characterize the bioadhesive matrix tablets employing various bioadhesive polymers for prolonged gastrointestinal absorption (Shamsuddin et al., 2006).

The present study was designed to study the capacity of hydrophilic polymers as release retardant and mucoadhesive agent in terms of dissolution study, retention time and data analysis.

\section{Materials and Methods}

\section{Chemicals}

Losartan potassium was kindly supplied by Globe Pharmaceuticals Limited, Noakhali, Bangladesh, as a gift sample and Hydroxy-propyl-methyl cellulose (HPMC), Methyl cellulose (MC), Sodium carboxymethyl cellulose (Na-CMC) were purchased from local market. Magnesium stearate and Lactose were obtained from Novo Pharmaceuticals Limited, Bangladesh. Solvents and all other chemicals used were of analytical grade.

Preparation of matrix tablets: Direct compression technique was employed for the preparation of Losartan potassium matrix tablets. Table 1 represents the 
formulation of matrix tablets with their codes such as F1 to F9. Amount of active ingredient was constant $(100 \mathrm{mg})$ in all cases. HPMC was used in the formulations indicated by $\mathrm{F} 1$ to $\mathrm{F} 3$ whereas $\mathrm{MC}$ was used in $\mathrm{F} 4$ to $\mathrm{F} 6$ and $\mathrm{Na}$ CMC was used in F7 to F9. Total weight of tablet was 355 mg. The required amount of active and polymers were weighed separately and mixed thoroughly. Matrix tablets were prepared by Perkin-Elmer (Germany) hydraulic press applying a compression force of 5 tons. Before compression surface of die and punch was lubricated with magnesium stearate. All the tablets were then stored in air tight containers at room temperature for further investigation.

In vitro dissolution studies: In vitro drug release studies were carried out in a paddle dissolution apparatus (Electrolab, India) equilibrated at $37 \pm 0.5^{\circ} \mathrm{C}$ at $50 \mathrm{rpm}$. The dissolution study was performed for 8 hours in $900 \mathrm{ml}$ of phosphate buffer ( $\mathrm{pH}$ 6.8) in triplicate. The dissolution samples were collected at 5,10,20,30,60 minutes and 2, $3,4,5,6,7,8 \mathrm{hr}$ and replaced with an equal volume of dissolution fluid $(5 \mathrm{m1})$. Sample solution was analyzed for Losartan potassium at $205 \mathrm{~nm}$ by an UV spectrophotometer (Shimadzu, Japan). The amount of drug present in the samples was calculated from the equation of standard curve.

Mucoadhesion test by in-vitro wash-off test: The mucoadhesive property of the prepared tablets was evaluated by an in vitro adhesion testing method known as wash off method (Lehr et al., 1990). Freshly excised pieces of intestinal mucosa $(3 \times 3$ inch $)$ from cow were mounted on the stainless steel slide, connected with a suitable support. Three tablets were placed onto wet rinsed tissue specimen and immediately thereafter the support was hung to the arm of the USP tablet disintegrating test machine. When the disintegrating machine was operated, the tissue specimen was given a slow, regular up-anddown movement in phosphate buffer $(\mathrm{pH} 6.8)$ at $37^{\circ} \mathrm{C}$.

Data treatment: The dissolution data was also fitted according to well-known exponential Korsmeyer-Peppas equation, which is often used to describe the drug release behavior from polymeric systems.

$\mathrm{M}_{\mathrm{t}} / \mathrm{M}_{\infty}=\mathrm{Kt}^{\mathrm{n}}$

Where $M_{t} / M_{\infty}$ is the fractional drug release at time $t$; $\mathrm{K}$ is a release rate constant incorporating structural and geometric characteristics of the tablet. A value of $n \leq 0.45$ indicates Fickian (case I) release; $>0.45$ but $<0.89$ for
non-Fickian (anomalous) release; and $>0.89$ indicates case II type of release (Ritger et al., 1987). From the above equation a value for different formulation was calculated to identify the drug release mechanism.

\section{Results and Discussion}

The serum half-life of Losartan potassium is 2 hours. It is well adsorbed through the length of gastrointestinal tract. Losartan potassium has a short elimination half-life, which encourages the drug to be formulated in sustained release dosage form. Due to its rapid elimination and narrow therapeutic index, this drug is indeed a suitable candidate to be formulated into sustained release dosage form (Reza et al., 2002).

Release kinetics: To know the mechanism of drug release from these formulations, the data were treated according to zero order (cumulative amount of drug released vs time), first-order (log cumulative percentage of drug remaining vs time), Higuchi (cumulative percentage of drug released vs square root of time) and Korsmeyer et al. (log cumulative percentage of drug released vs log time) pattern (Reddy et al., 2003). When the data were plotted according to zero-order all formulations except F5 (0.861) and F1 (0.709) showed linearity $\left(\mathrm{R}^{2}>0.94\right)$. In case of Higuchi model, a fair linearity was obtained $\left(\mathrm{R}^{2}>0.90\right)$ except F7 (0.896) and F1 (0.826). To confirm the drug release mechanism, the data were fitted into Korsmeyer's equation. The $\mathrm{n}$ (slope of Korsmeyer equation) value ranging from $0.207-0.683$ for al formulations was estimated. F1 to F4 exhibited Fickian type drug release and on the other hand F5 to F9 exhibited Non-Fickian type drug release.

Effect of HPMC on drug release: HPMC is water soluble as it contains free hydroxyl group. The ability of HPMC to prevent burst release was also claimed (Lee et al., 1999). From the figure 1(A), after $1^{\text {st }} 5 \mathrm{~min}$ the amount of released drugs are 19.36, 15.27 and $12.62 \%$ for F1, F2, $\mathrm{F} 3$, respectively whereas the amount of released drugs after $8 \mathrm{hrs}$ are $67.38,80.93$ and $74.61 \%$. These formulations contain $100 \mathrm{mg}$ drug and $50 \mathrm{mg}, 100 \mathrm{mg}$, $150 \mathrm{mg}$ HPMC, respectively. F1 gave better drug release profile $(67.38 \%$ after 8 hours) although it contains less amount of polymer than other formulations. The regression coefficients of Higuchi model are 0.826, 0.954, 0.987 for F1, F2, F3 respectively. HPMC based formulations show Fickian type release. Figure 2 (A) 
shows the first order release pattern for the formulated tablets.

Effect of MC on drug release: $\mathrm{MC}$ is a water soluble compound where the hydroxyl group of Cellulose is replaced by methyl group. From the figure 1(B), after $1^{\text {st }} 5$ min the amount of released drugs were 9.01, 8.10 and $6.66 \%$, respectively for F4, F5, F6 whereas the amount of released drugs after $8 \mathrm{hrs}$ are $69.48,68.28$ and $64.06 \%$. These formulations contain $100 \mathrm{mg}$ drug and $50 \mathrm{mg}, 100$ $\mathrm{mg}, 150 \mathrm{mg} \mathrm{MC}$, respectively. Methyl cellulose showed excellent linearity as the release rate decreases with an increase in polymer content. The regression coefficients of Higuchi model are 0.94, 0.953, 0.974 for F4, F5 and F6, respectively. F4 shows Fickian type release and F5 and F6 show Non-Fickian type release. First order release data has been plotted in figure 2 (B).

Effect of Na-CMC on drug release: $\mathrm{Na}-\mathrm{CMC}$ is most water soluble as it is a salt form of CMC. After $1^{\text {st }} 5 \mathrm{~min}$ the amount of released drugs were 10.51, 5.88 and $3.11 \%$, respectively for F7, F8 and F9 whereas the amount of released drugs after $8 \mathrm{hrs}$ were $78.13,70.30$ and $81.74 \%$. These formulations contain $100 \mathrm{mg}$ drug and $50 \mathrm{mg}, 100$ $\mathrm{mg}, 150 \mathrm{mg} \mathrm{Na}-\mathrm{CMC}$, respectively. The regression coefficients of Higuchi model are 0.896, 0.962, 0.909 for F7, F8 and F9, respectively. F8 shows good release pattern as required for sustained release. $\mathrm{Na}-\mathrm{CMC}$ based formulations show Non-Fickian type release. The use of $\mathrm{Na}-\mathrm{CMC}$ in bio-adhesive drug delivery system has been reported earlier (Rhodes et al., 1994).

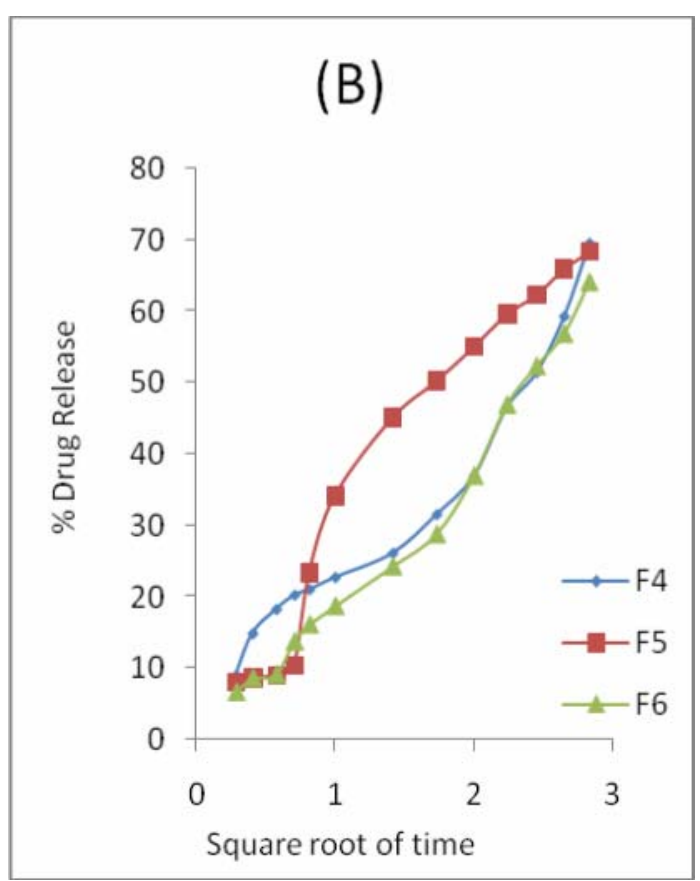

(A): HPMC based matrix tablets, (B): MC based matrix tablets.

Figure 1. Higuchi release profile of matrix tablets of Losartan potassium.

Table 1. Formulations of Losartan potassium matrix tablets.

\begin{tabular}{lccccccccc}
\hline Ingredients (mg) & F1 & F2 & F3 & F4 & F5 & F6 & F7 & F8 & F9 \\
\hline Losartan Potassim & 100 & 100 & 100 & 100 & 100 & 100 & 100 & 100 & 100 \\
HPMC & 50 & 100 & 150 & - & - & - & - & - & - \\
MC & - & - & - & 50 & 100 & 150 & - & - & - \\
Na CMC & - & - & - & - & - & - & 50 & 100 & 150 \\
Lactose & 200 & 150 & 100 & 200 & 150 & 100 & 200 & 150 & 100 \\
Mg stearate & 5 & 5 & 5 & 5 & 5 & 5 & 5 & 5 & 5 \\
Total & 355 & 355 & 355 & 355 & 355 & 355 & 355 & 355 & 355 \\
\hline
\end{tabular}



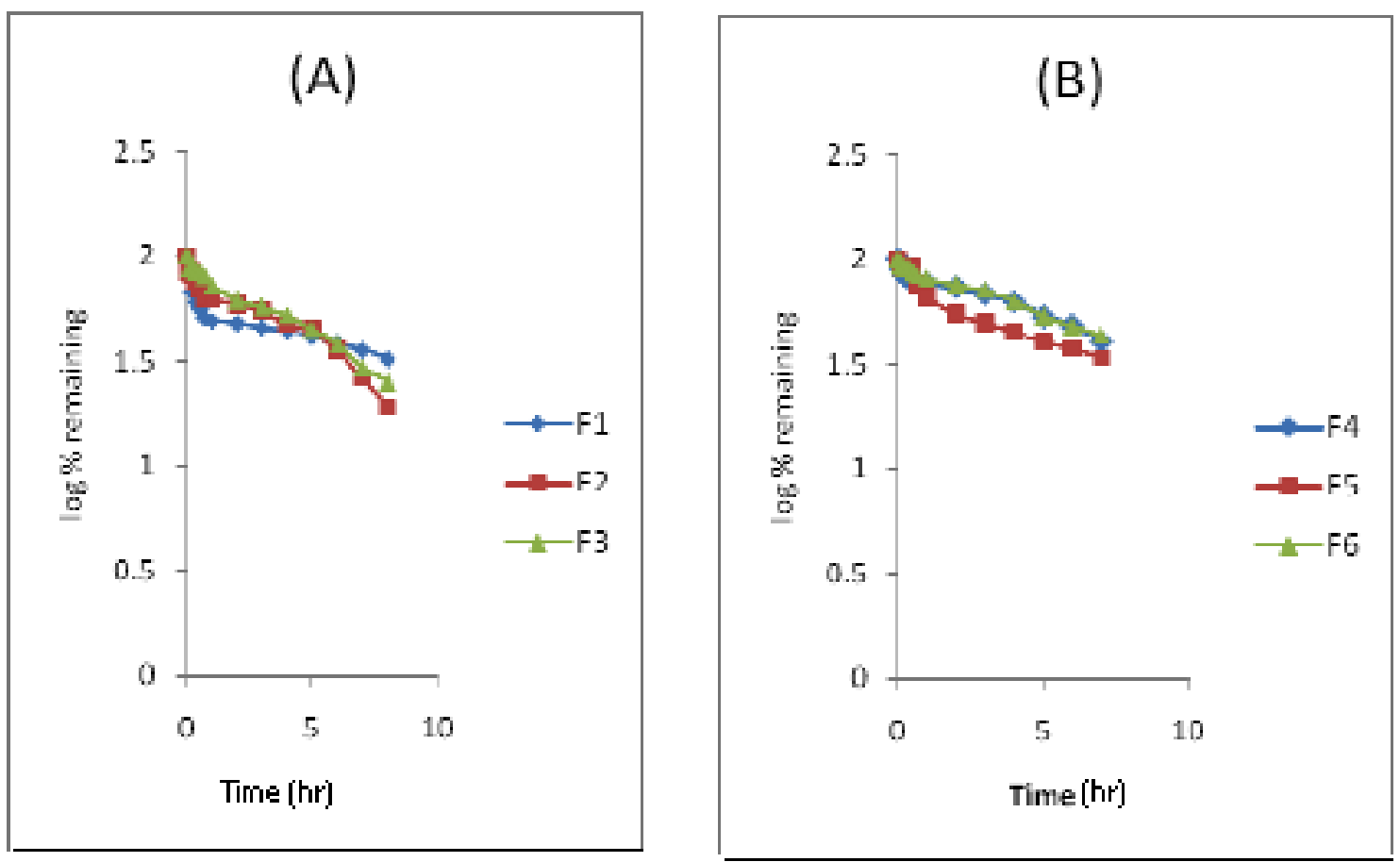

(A): HPMC based matrix tablets, (B): MC based matrix tablets.

Figure 2. First order release profile of matrix tablets of Losartan potassium.

Mucoadhesive property of HPMC: In case of HPMC, the mucoadhesive property increases with an increase in polymer content. All the three formulations of HPMC showed poor mucoadhesive property. F1 was detached after $35 \mathrm{~min}, \mathrm{~F} 2$ was detached after $69 \mathrm{~min}, \mathrm{~F} 3$ showed slight good result than the other two formulations and the time was $85 \mathrm{~min}$.

Mucoadhesive property of MC: The more MC used in the formulation, the more mucoadhesive property it shows. Methyl cellulose based formulations showed good mucoadhesive property. F4 detached from mucous membrane after 117 min from the time of attachment. F5 detached at $142 \mathrm{~min}$. And F6 was attached with mucous membrane for $240 \mathrm{~min}$.

Mucoadhesive property of Na-CMC: The mucoadhesive property increases with an increase in polymer content. Increasing the polymer amount may provide more adhesive sites and polymer chains for interpenetration with mucin, resulting in the augmentation of bioadhesive strength. Na-CMC showed better mucoadhesive property as F7 took 92 min to be detached, F8 took $182 \mathrm{~min}$ where F9 took $240 \mathrm{~min}$.

\section{Conclusion}

Studies for Mucoadhesive properly and subsequent release profile were performed on matrix tablets of Losartan potassium. From pharmaceutical and biopharmaceutical viewpoint, it can be said that the most potential polymer is that one which possesses strong mucoadhesive property, sufficient release retarding capacity and provides a release profile that meets USP specification. $\mathrm{MC}$ have been proved eligible to be used in mucoadhesive sustained release dosage form, whereas $\mathrm{Na}$ CMC possesses only mucoadhesive property and HPMC has less mucoadhesive property and sustained release property. However, further investigation should be carried out to establish stability and reproducibility of this dosage form. Moreover, other experiments should be explored to find out the real mucoadhesion in animals. Finally, in vitro in vivo correlation should be performed to assess the efficacy of this mucoadhesive dosage form.

\section{References}

Hannah, B. 2004. Nobel bioadhesive formulations in drug delivery. Presented at British pharmaceutical conference on drug delivery to upper GI tract, particularly to esophagus. Medicines research unit, Aston University, Birmingham, B47ET, UK. 
Lee, B.J., Ryu, S.G. and Cui, J.H. 1999. Formulation and release characteristics of hydroxypropylmethylcellulose matrix tablet containing melatonin. Drug. Dev. Ind. Pharm. 25, 493-501.

Lehr, C.M., Bowstra, J.A., Tukker, J.J. and Junginer, H.E. 1990. Intestinal transit of bioadhesive microspheres in an in-situ loop in rat. J. Contr. Rel. 13, 51-62.

Reddy, R., Mutalik, S. and Reddy S. 2003. Once-daily sustainedrelease matrix tablets of nicorandil: formulation and in vitro evaluation, AAPS Pharm. Sci. Tech, 4, 61.

Remington, 2005. The Science and Practice of Pharmacy, BI Publications, $21^{\text {st }}$ edn. 939.

Reza, S., Quadir, M.A. and Haider, S.S. 2002. Development of theophylline sustained release dosage form based on kollidon SR. Pak. J. Pharm. Sci. 15, 63-70.

Rhodes, C.T., Jimenez-castellanos M.R. and Zia, H. 1994. Design and testing in-vitro of a bioadhesive floating drug delivery system for oral application. Int. J. Pharm. 105, 65-70.
Ritger, P.L. and Peppas, N.A. 1987. A simple equation for description of solute release II. Fickian and anomalous release for sweallable devices. J. Contr. Rel. 5, 37-42.

Schachter, H. and Williams, D. 1982.Biosynthesis of mucous glycoproteins. Adv. Exp. Med. Biol. 144. 3.

Shamsuddin, A.P., Khan, J.R., Chowdhury, J.A. and Reza, S. 2006. Studies on bio-adhesion of matrix tablets: Release profile of theophylline anhydrous. Dhaka Univ. J. Pharm. Sci. 5, 33-37.

Vyas, S.P. and Khar, R.K. 2002. Controlled Drug Delivery, $1^{\text {st }}$ edn. Jain, M. K., Delhi, 199.

Wilson, C.G. and Washington, N. 1989. Biological barrier to drug absorption. In: Physiological Pharmaceutics. Ellis Harwood, Chickestar, p. 47. 